\title{
Recent follow-up observations of GRBs in the very high energy band with the MAGIC telescopes
}

\section{A. Carosi ${ }^{* a}$, L.A. Antonelli ${ }^{a}$, J. Becerra González ${ }^{b}$, A. Bertic ${ }^{c}$, S. Covino ${ }^{a}$, M. Garczarczyk $^{d}$, M. Gaug ${ }^{e}$, S. Lombardi ${ }^{a}$, F. Longo ${ }^{c}$, E. Moretti ${ }^{f}$, K. Noda ${ }^{f}$, L. Nogués ${ }^{g}$, M. Peresano ${ }^{c}$, M. Persic ${ }^{c}$, M. Teshima ${ }^{f}$, T. Toyama $^{f}$, M. Will ${ }^{g}$ for the MAGIC Collaboration}

E-mail: alessandro.carosi@oa-roma.inaf.it

a INAF, National Institute for Astrophysics, I-00136 Rome, Italy;

${ }^{b}$ NASA Goddard Space Flight Center, Greenbelt, MD 207771, USA

${ }^{c}$ Universitá di Udine, and INFN Trieste, I-33100 Udine, Italy;

${ }^{d}$ Deutsches Elektronen-Synchrotron (DESY), D-15738 Zeuthen, Germany;

${ }^{e}$ Unitat de Física de les Radiacions, Departament de Física, and CERES-IEEC, Universitat Autonoma de Barcelona, E-08193 Bellaterra, Spain

${ }^{f}$ Max-Planck-Institut für Physik, D-80805 München, Germany;

${ }^{g}$ IFAE, Edifici Cn., Campus UAB, E-08193 Bellaterra, Spain;

Gamma ray burst (GRBs) are primary targets for all modern IACT telescopes. The MAGIC collaboration has identified the detection of GRBs in the VHE regime as one of its multi-year key observational programs (KOP). However, the transient and unpredictable nature of GRBs makes pointing and rapid follow-up observations to observe the prompt emission phase difficult for large ground-based Cherenkov facilities. Thanks to its fast pointing speed and low-energy sensitivity, MAGIC is particularly well suited for GRB studies in the VHE range below 100 $\mathrm{GeV}$ during the prompt-to-early afterglow phase.

Since the beginning of operation in 2004-2005, MAGIC has performed 74 follow-up observations that, to date, have yielded no significant detections. However, in the last two years, the upgrade of the MAGIC system and an improved GRB observation procedure has made possible follow-up of GRBs within 100s after the event onset, thus opening a new phase in the study of GRBs with MAGIC. In this contribution, I report on the achieved performance and some characteristics of the GRBs observed with MAGIC, with particular focus on those observed after the performance improvements in 2013.

The 34th International Cosmic Ray Conference,

30 July- 6 August, 2015

The Hague, The Netherlands

\footnotetext{
* Speaker.
} 


\section{Introduction}

Since almost 50 years from their discovery, the physical origin of GRB represent one of the most enigmatic open questions of astrophysics (see [1] for a recent review). These short and intense pulses of gamma-ray detected at an average rate of $\sim 1$ per day, are the targets of large observational programs in different energy bands by both ground-based and space-based instruments. In the last decade, our comprehension of GRB physics has significantly evolved, mainly thanks to the simultaneous availability of dedicated instruments on-board the Swift and Fermi satellites and to the improving multi-wavelength capabilities for follow-up observation by ground-based facilities. The wealth of broad-band data extends from the radio band up to the gamma energy range. While emission from these objects is mainly observed in the sub-MeV band, the observations of FermiLAT have shown that many GRBs radiate significantly above a few tens of $\mathrm{GeV}$ (up to $94 \mathrm{GeV}$ as in the remarkable case of GRB 130427). Thus, GeV emission has definitively been proven to be a relatively common feature likely for the large majority of the events although only for the brightest $\sim 5 \%$ of the GBM-detected events, the detection is achieved with current instruments [2]. On the other hand, such an increasing number of $\mathrm{GeV}$ bursts does not combine with an appropriate theoretical understanding of these events. In the majority of LAT-detected GRB, GeV emission occurs with a significant delay and with a longer-lasting activity with respect to the $\mathrm{MeV}$ and sub-MeV emission. From the spectral point of view, GeV GRBs can usually be well interpreted using the well-known phenomenological Band function [3] (two smoothly-connected power law segments) extending up to the $\mathrm{GeV}$ band although, in some cases, a second harder emission component is clearly detectable and overlapped to the Band emission that still dominate the GBM band (see e.g., [4]). The resulting poor interpretative framework points out to the importance of the detection of a possible VHE signal above $100 \mathrm{GeV}$ in order to distinguish between different theoretical models.

The MAGIC telescopes are particularly suited for GRB study thanks to the fast repositioning speed and the low energy threshold that reduce the flux attenuation by pair production with the lower energy (optical/IR) photons of the diffuse extragalactic background light (EBL). Several results about GRB follow-up have been already discussed in literature by MAGIC $[5,6,7]$ and other IACT [8]. In this proceeding, we report the performance and results achieved by MAGIC during the last $\sim 2$ years, after the implementation of an improved GRB automatic observation procedure. This procedure permits to start data-taking even within few tens of seconds from GRB announcement. Unfortunately, no gamma-ray signal was detected.

\section{GRB physiscs in the VHE band}

Within the framework of relativistic blast-wave models, possible emission in the $>100 \mathrm{GeV}$ band by synchrotron-self-Compton (SSC) mechanism in afterglows have been predicted and discussed by MAGIC in relation to the obtained upper limits [6,7]. This model, although frequently used in the Swift and Fermi GRB phenomenology description due to its simplicity, usually fails in the description of the observed features in $\mathrm{HE}$ band such as the delayed $\mathrm{MeV}-\mathrm{GeV}$ emission [9]. 


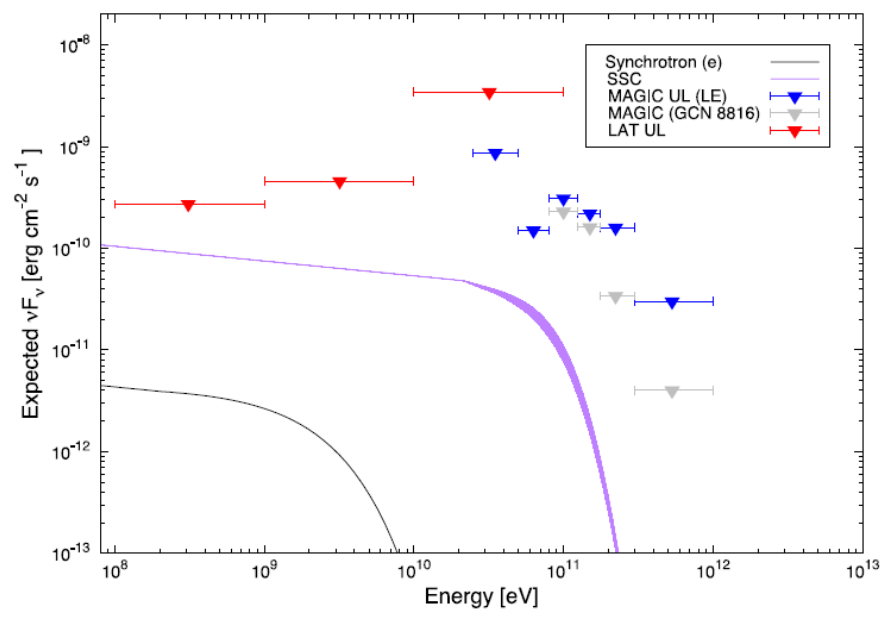

Figure 1: Modelled emission during the afterglow of GRB 090102. Blue triangles are 95\% CL ULs derived by MAGIC and optimized for low-energy analysis. For comparison, the regular energy range MAGIC ULs are also reported in light grey. The red triangles report the Fermi-LAT 95\% CL ULs. The purple and black curves depict the expected energy flux according to the GRB afterglow model reported in [7] for the SSC and electron synchrotron processes respectively. The shaded region shows the uncertainty in the EBL attenuation according to [10]. From [7].

Alternative processes proposed, as a natural explanation of the delay in the emission of the HE and VHE components, the separation between an inner source of soft photons from an outer region where they are up-scattered to GeV energies [11] [12]. Such a model can be generally considered as different variations of an External Inverse Compton (EIC) mechanism which has been taken into account for the explanation of the VHE emission in blazars. The seed photons are produced by delayed activity of the central engine in the form of X-ray flares or re-brightening (as an X-ray plateau observed in a large number of events). When seed photons reach the blast wave, they can be up-scattered via Inverse Compton mechanism by shock accelerated electrons producing a MeV$\mathrm{GeV}$ component. Other mechanism envisions the possibility that the seed photons derive from the modified thermal emission from a optically thin "photosphere" within GRB outflow [12]. The occurrence of energy dissipation processes both below and above the photosphere (like internal shocks and interaction with stellar structure envelope) could modify the normal black-body emission bringing to a Band-like spectrum peaked at $\sim 1 \mathrm{MeV}$. The observational distinction of this large variety of models may be crucial for clarifying the physics of the early afterglow in general. Furthermore, VHE observations of GRBs with high sensitivity can play a decisive role in solving unresolved issues as determine the bulk Lorentz factor of the outflow through internal $\gamma \gamma$ absorption features or reveal the possible hadronic signatures associated with production of UHECRs and neutrinos.

\section{The MAGIC telescopes and the GRB follow-up}

The MAGIC system consists of two $17 \mathrm{~m}$ diameter Imaging Atmospheric Cherenkov Telescopes (IACTs) located at the Roque de los Muchachos observatory $\left(28.8^{\circ} \mathrm{N}, 17.8^{\circ} \mathrm{W}, 2200 \mathrm{~m}\right.$ 
a.s.1.), on the Canary Island of La Palma. The MAGIC system is currently carrying out stereoscopic observations with a sensitivity of $<0.7 \%$ of the Crab Nebula flux for energies above $\sim 220 \mathrm{GeV}$ in $50 \mathrm{~h}$ of observation, and a trigger energy threshold of $50 \mathrm{GeV}$ at zenith. Since the beginning of its operation, MAGIC is able to react to GRB alerts thanks to a dedicated alert system receiving the external trigger provided by the GRB Coordinate Network $\left(\mathrm{GCN}^{1}\right)$ trough TCP/IP socket.

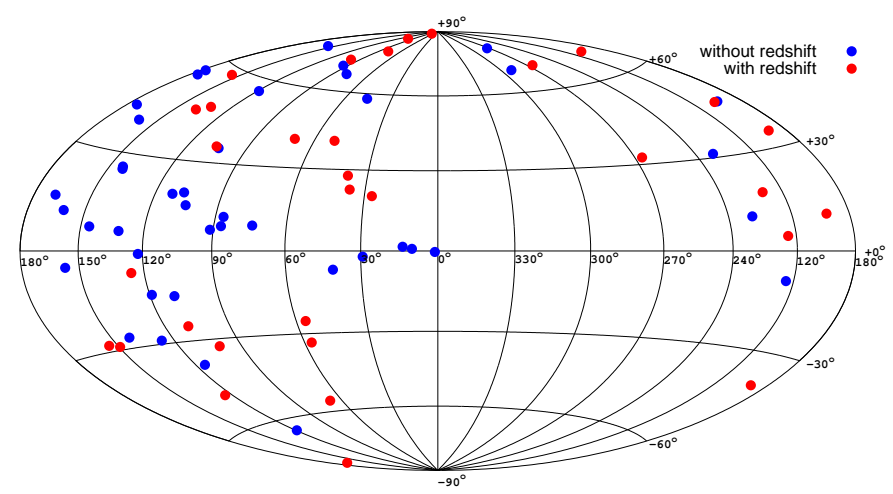

Figure 2: Galactic aitoff projection of the positions of the GRBs followed-up by the MAGIC telescopes.

The observation of GRBs has the highest priority among MAGIC observed targets: as soon as the alert is received and validated by the alert system, according to some pre-defined criteria, the event's coordinates are passed to the central control of the telescopes. A fully automated procedure takes only few seconds to stop the ongoing observation, point to GRB position and start taking data. Thanks to their lightweight structures based on carbon fiber tubes, the MAGIC telescopes are able to point to GRB coordinates using dedicated fast-slewing movements within few tens of seconds after the alert is received. This implies a remarkable re-pointing speed of around $7 \% \mathrm{sec}$ in GRB mode in both zenith and azimuth ${ }^{2}$. To prevent possible failures and problems during the initial phases of GRB follow-up, at the beginning of 2013, a new automatic procedure has been implemented: in case of GRB alert, the data acquisition (DAQ [14]) is not stopped and restarted once the GRB position is reached. DAQ continues taking data during telescopes slewing guaranteeing a reduction in the number of failures as well as a faster start of data taking. Furthermore, observations are carried on in wobble mode. Historically, GRB were observed in ON/OFF mode that marginally accounts for a better instrument sensitivity but with an increasing uncertainties in the background evaluation. Based on observational constrains, the GRB observation is carried on for a maximum observation time of $4 \mathrm{~h}$ after the prompt emission. This guarantees the follow-up of the event up to the late afterglow emission phase.

The main characteristics of the GRBs observed after the above-mentioned MAGIC upgrade are reported in Tab. 1

\footnotetext{
${ }^{1}$ http://gcn.gsfc.nasa.gov/

${ }^{2}$ The normal pointing speed for MAGIC is around $3 \%$ s.
} 

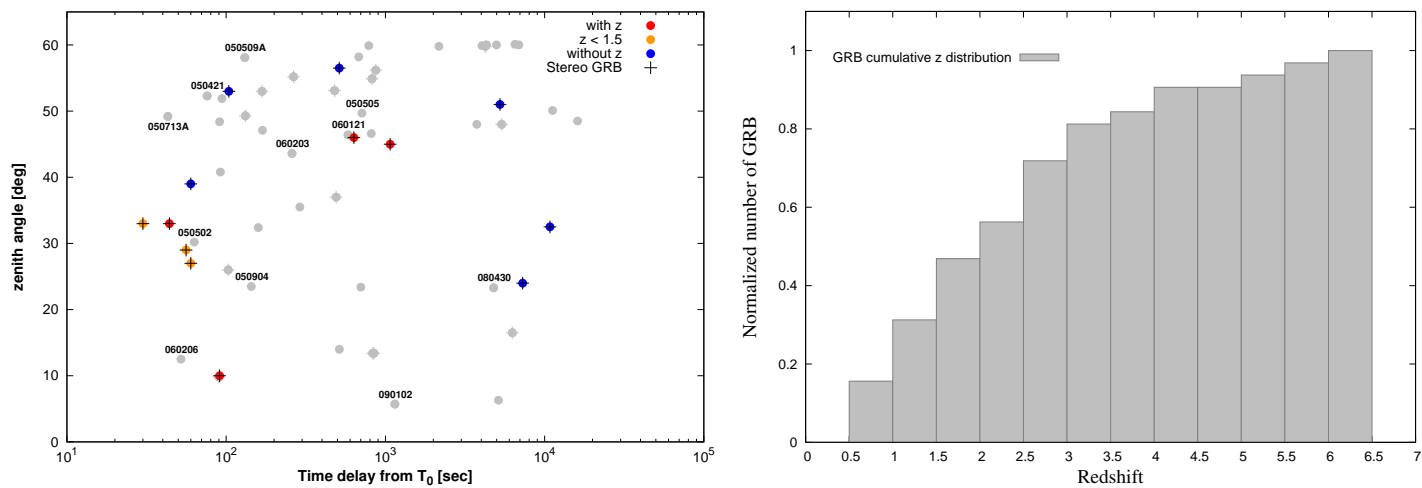

Figure 3: Left: Zenith angle vs time delay at the moment of the GRB observation performed by MAGIC. The achieved results of the new automatic procedure are pointed out through the fast population of the plot in the $\simeq 100 \mathrm{~s}$ region. Light gray points are events observed with previous procedure or during the mono phase while the labeled GRBs have been target of past dedicated publications. Right: redshift cumulative distribution of the GRBs observed by MAGIC since 2004: about 50\% of the known-redshift GRBs have a redshift below 2 .

\begin{tabular}{|l|ccccccc|}
\hline & $\begin{array}{c}\mathrm{T}_{0} \\
{[\mathrm{UTC}]}\end{array}$ & $\begin{array}{c}\mathrm{T}_{90} \\
{[\mathrm{~s}]}\end{array}$ & $\mathrm{z}$ & $\begin{array}{c}\text { Alert time } \\
{[\mathrm{UTC}]}\end{array}$ & $\begin{array}{c}\text { Start time } \\
{[\mathrm{UTC}]}\end{array}$ & $\begin{array}{c}\text { Zenith } \\
{[\mathrm{deg} .]}\end{array}$ & $\begin{array}{c}\text { Delay } \\
{[\mathrm{sec} .]}\end{array}$ \\
\hline GRB 130502A & $17: 50: 30$ & 3 & - & $17: 53: 59$ & $20: 57: 03$ & $33-40$ & $11247^{*}$ \\
GRB 130504 & $02: 05: 34$ & 50 & - & $02: 06: 01$ & $02: 13: 09$ & $56-45$ & 455 \\
GRB 130606 & $21: 04: 39$ & & 5.9 & $21: 05: 02$ & $21: 15: 13$ & $46-2$ & $634^{*}$ \\
GRB 130612A & $03: 22: 22$ & 5.6 & 2.0 & $03: 22: 46$ & $03: 23: 08$ & $33-53$ & 48 \\
GRB 130701 & $04: 17: 43$ & 4.4 & 1.16 & $04: 18: 02$ & $04: 18: 31$ & $27-16$ & 48 \\
GRB 131030 & $20: 56: 19$ & 41 & 1.3 & $20: 56: 34$ & $20: 56: 45$ & $33-40$ & 26 \\
GRB 140430 & $20: 33: 36$ & 173.6 & 1.60 & $20: 33: 51$ & $20: 52: 06$ & $45-54$ & $1110^{*}$ \\
GRB 140709 & $01: 13: 41$ & 98.6 & - & $01: 14: 02$ & $03: 17: 15$ & $25-37$ & $7414^{*}$ \\
GRB 140930 & $19: 41: 42$ & 0.84 & - & $19: 42: 02$ & $21: 10: 05$ & $51-18$ & $5243 *$ \\
GRB 141026 & $02: 36: 51$ & 146 & 3.35 & $02: 38: 09$ & $02: 38: 27$ & $10-50$ & 96 \\
GRB 141220 & $06: 02: 52$ & 7.21 & 1.32 & $06: 03: 17$ & $06: 03: 47$ & $29-19$ & 55 \\
GRB 150428A & $01: 30: 40$ & 53.2 & - & $01: 31: 43$ & $01: 32: 10$ & $37-54$ & 90 \\
GRB 150428B & $03: 12: 03$ & 130.9 & - & $03: 12: 38$ & $03: 13: 02$ & $50-27$ & 59 \\
\hline
\end{tabular}

Table 1: GRBs observed by the MAGIC telescopes with the improved automatic procedure. Columns represent respectively: the GRB name, the satellite trigger time, the duration of the event at X-rays $\left(\mathrm{T}_{90}\right)$, the GRB redshift, the start time of MAGIC observation, the zenith angle range between the beginning and the end of the follow-up and the overall delay between the beginning of data taking and the burst onset. Entries marked with $(*)$ are the events observed at late time due to observational constraints.

\section{Data Analysis}

The preliminary data analysis was performed using the MAGIC analysis package MARS [15]. After the calibration of the single events recorded by each telescope, the data were processed with the standard cleaning process described in [13]. For the gamma/hadron separation 
and gamma-direction estimation, a multivariate method based on a random forest (RF) algorithm was applied [16]. This algorithm employs some Cherenkov image parameters [17] to compute a gamma/hadron discriminator called hadronness by comparison with Monte Carlo gamma-ray simulations. The energy of the events was estimated by averaging individual energy estimators for both telescopes based on look-up tables. The detection of the possible gamma-ray signal is achieved through the so-called $\theta^{2}$ plot, i.e. the comparison between the distributions of the square angular distance between the reconstructed position of the source and its nominal position in the signal and background regions for energies above the threshold. The significance of the signal is evaluated using single cuts in hadronness and $\theta^{2}$ and according to Eq. 17 of [18].

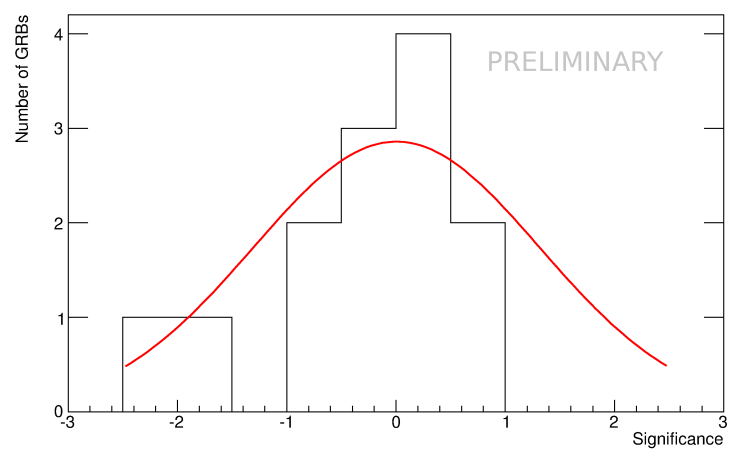

Figure 4: Significance distribution of the GRBs reported in Tab. 1. The resulting fit is compatible with a gaussian distribution centered at zero and variance one.

The performed analysis has shown a $\theta^{2}$ distribution from the different GRB data sets compatible with the distribution expected from background hadronic events, implying no significant VHE gamma-ray signal for any of the observed GRB over the duration of MAGIC follow-up.

\section{GRBs of special interest}
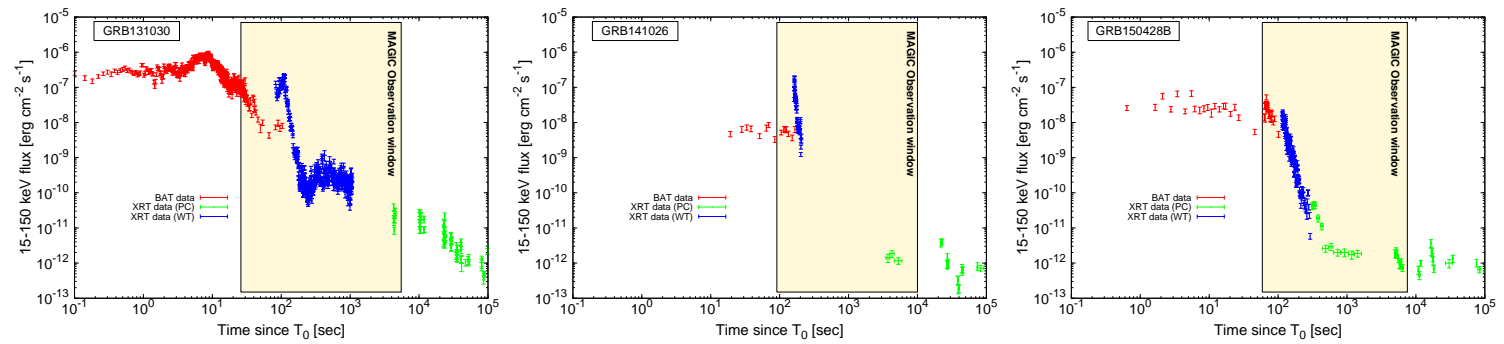

Figure 5: Combined BAT and XRT light curve for GRB 131030, GRB 141026 and GRB 150428. XRT PC and WT mode (blue and green points respectively) and BAT data (red points) are obtained using the automatic analysis of Swift data (http://www.swift.ac.uk/burst_analyser). The corresponding MAGIC observation windows are also plotted.

In some cases, MAGIC was able to start the follow up already during the prompt emission phase. The light curves corresponding to these GRBs are depicted in Fig. 5 with the MAGIC observational window: 
- GRB 131030 was triggered and located at 20:56:19 UT by Swift. The BAT light curve shows two overlapping peaks starting at $\mathrm{T}_{0}-8 \mathrm{~s}$, peaking at $\mathrm{T}_{0}+10 \mathrm{~s}$, and ending at around $\mathrm{T}_{0}+180$ $\mathrm{s}$ with a low-level tail out to at least $\mathrm{T}_{0}+800 \mathrm{~s}$ [19]. The event was a relatively low redshift GRB $(z=1.29)$ and MAGIC was able to point at events coordinates in less then $30 \mathrm{~s}$ with respect to $\mathrm{T}_{0}$. Unfortunately, the first $\sim 30$ minutes of the observation were affected by adverse atmospheric conditions.

- GRB 141026 was triggered and located by Swift at 02:36:51 UT. The BAT light curve shows a faint pulse starting at $T_{0}+15 \mathrm{~s}$, and ending around $T_{0}+180 \mathrm{~s}$. The best fit decay curve is achieved by a simple power-law function with a time-averaged spectral index of $2.34 \pm$ 0.19 [20]. MAGIC started to observe the event $\sim 90 \mathrm{~s}$ after the alert at a very good zenith angle $\left(10^{\circ}\right)$. However, the measured redshift $(z=3.35)$ complicates the detection of a VHE emission component.

- GRB 150428B was triggered and located by Swift at 03:12:03 UT. The BAT light curve shows three separate peaks distributed between $T_{0}-40 \mathrm{~s}$ and $T_{0}+120 \mathrm{~s}$. In this time slot, the spectrum is fitted with a power law with an exponential cutoff giving a photon index of $1.0 \pm 0.5$ and a peak energy of $55 \pm 10 \mathrm{keV}$ [21]. MAGIC reacted to the alert pointing at GRB coordinates after about 1 minute but at relatively high zenith. No redshift measurements are available.

\section{Conclusion}

The MAGIC telescopes have proven to be well suited for GRB follow-up observations in the VHE regime thanks to its low-energy threshold and its remarkable fast pointing capability. However, no VHE emission has been detected neither during the afterglow nor during the few prompt emission phases that could be followed. The non-detection of VHE gamma-rays with MAGIC does not necessarily imply a physical lack of VHE photons from the observed events as ground-based observation were often affected by adverse observational conditions and, as a matter of fact, only few events were observed during good conditions. The development of a new observational procedure has recently improved the performance of MAGIC for GRB follow-up observations. Additional efforts to improve the analysis in the very low energy band, as well as further improvements in the observation strategy are being investigated. The results of the final analysis of the events reported here will be the target of a dedicated forthcoming publication.

\section{Acknowledgments}

We would like to thank the Instituto de Astrofisica de Canarias for the excellent working conditions at the Observatorio del Roque de los Muchachos in La Palma. The financial support of the German BMBF and MPG, the Italian INFN and INAF, the Swiss National Fund SNF, the ERDF under the Spanish MINECO (FPA2012-39502) and MECD (FPU13/00618), and the Japanese JSPS and MEXT is gratefully acknowledged. This work was also supported by the Centro de Excelencia Severo Ochoa SEV-2012-0234, CPAN CSD2007-00042, and MultiDark CSD2009-00064 projects of the Spanish Consolider-Ingenio 2010 programme, by grant 268740 of the Academy of Finland, by the Croatian Science Foundation (HrZZ) Project 09/176 and the University of Rijeka Project 13.12.1.3.02, by the DFG Collaborative Research Centers SFB823/C4 and SFB876/C3, and by the Polish MNiSzW grant 745/N-HESS-MAGIC/2010/0. 


\section{References}

[1] Mészáros \& Rees, Gamma Ray Burt, arXiv e-prints 1401.3012 (2014) - To be published in "General Relativity and Gravitation: A Centennial Perspective"

[2] Granot, J., Highlights from Fermi GRB observations arXiv e-prints 1003.2452 (2010) Proceedings of "The Shocking Universe - Gamma-Ray Bursts and High Energy Shock phenomena"

[3] Band, D., Matteson, J., Ford, L. et al., BATSE observations of gamma-ray burst spectra. I - Spectral diversity, ApJ 413 (1993) 281

[4] Ackermann, M. et al., Fermi observations of GRB 090510: a short-hard Gamma-Ray Burst with an aitional, hard power-law component form $10 \mathrm{keV}$ to GeV energies ApJ 716 (2010) 1178

[5] Albert, J. et al. (MAGIC Collaboration), MAGIC Upper Limits on the Very High Energy Emission from Gamma-Ray Bursts ApJ 667 (2007) 358

[6] Aleksić, J. et al. (MAGIC Collaboration), MAGIC observation of GRB080430 afterglow A\&A 517 (2010) 5

[7] Aleksić, J. et al. (MAGIC Collaboration), MAGIC upper limits on the GRB 090102 afterglow MNRAS 437 (2014) 3103

[8] Acciari, V. et al. (VERITAS collaboration), VERITAS observations of gamma-ray bursts detected by swift, ApJ 743 (2011) 62

[9] Asano, K. \& Meszaros, P. Spectral-Temporal Simulations of Internal Dissipation Models of Gamma-Ray Bursts, ApJ 739 (2011) 103

[10] Domínguez, A, Primack, J. R., Rosario, D. J. et al., Extragalactic background light inferred from AEGIS galaxy-SED-type fractions, MNRAS, 410 (2011) 2556

[11] Fan, Y. Piran, T., Narayan, R. et al., High-energy afterglow emission from gamma-ray bursts, MNRAS 384 (2008) 1483

[12] Toma, K., Xue-Feng W., \& Meszaros, P. An up-scattered cocoon emission model of Gamma-Ray Burst high-energy lags, ApJ 707 (2011) 1404

[13] Aleksić, J. et al. (MAGIC collaboration), The major upgrade of the MAGIC telescopes, Astroparticle Physics 72 (2016) 76

[14] Tescaro, D., Lopez-Oramas, A., Moralejo, A. et al., The MAGIC telescopes DAQ software and the on-the-fly online analysis client In Proc. of the 33st ICRC, Rio de Janeiro, Brasil (2013)

[15] R. Zanin et al., MARS, the MAGIC Analysis and Reconstruction Software, In Proc. of the 33st International Cosmic Ray Conference, Rio de Janeiro, Brasil (2013)

[16] Albert, J., Aliu, E., Anderhub, H., et al., Implementation of the Random Forest Method for the Imaging Atmospheric Cherenkov Telescope MAGIC, Nucl. Instrum. Meth., A588 (2008) 424

[17] Hillas, A. M. 1985, Cherenkov light images of EAS produced by primary gamma, In Proc. of 19th, International Cosmic Ray Conference, La Jolla, (1985)

[18] Li, T.-P., \& Ma, Y.-Q., Analysis methods for results in gamma-ray astronomy, ApJ 272 (1983) 317

[19] Barthelmy, S. D., Baumgartner, W. H. et al. GRB 131030A: Swift-BAT refined analysis GCN 15456 (2013)

[20] Stamatikos, M., Barthelmy, S. D. et al. GRB 141026A: Swift-BAT refined analysis GCN 16960 (2014)

[21] Cummings, J. R., Barthelmy, S. D. et al. GRB 150428B: Swift-BAT refined analysis GCN 17776 (2015) 